\title{
Acknowledgement of Reviewers
}

The Managing Editor, Prof. Michael Rycroft, members of the Editorial Board and all members of staff at Springer who are connected with our journal are most grateful to the reviewers named below for their hard work and diligence when refereeing papers submitted for publication. Their assistance in raising the standard of the papers published in Surveys in Geophysics is immense, and is greatly appreciated.
N. Abrahamsen
Y. Gu
P. Queralt
J. Albert
G. Heinson
V. Rapprich
K. Aplin
S. Hekimoglu
A. Revil
D. Avdeev
H. Hill
H. Russchenberg
J.-P. Avouac
A. Hoerdt
M. C. Santos
P. Banerjee
C. Houser
I. Sass
M. Berber
H. Jiang
G. Schwarz
A. Berne
R. Krier
M. Sorensen
T.A. Blix
G. Laske
P. Soupios
M. Bos
A. $\mathrm{Li}$
K. Strack
N. Cassidy
M. Cho
M. Malinowski
M. Thorne
V. Mushtak
V. Tong
S. Clandillon
B. Nagarajan
D. Tsoulis
M. Craymer
F. Napolitano
J. Tyburczy
E. Debayle
P. Novak
L. Vinnik
A. Deuss
P. Ogunbona
H. Wenbao
D. Dobson
Y. Omura
$\mathrm{Y}$. $\mathrm{Wu}$
M. A. Endres
G. Ottavianelli
J. Xia
W. Featherstone
N. Pavlis
H. Xiangyun
J. Gaspar-Escribano
J. Pek
H. Yang
W. Geissler
N. Penna
Y. Yang
R. Goldberg
S. Petrovic
X. Yuan 